\title{
Creación de una lente de contacto basándose en la investigación del mercado y sus aplicaciones como método de aprendizaje.
}

\section{Contact lens design based on market research and its applications as a learning method.}

MARÍA JosÉ BAUTISTA LLAMAS

https://orcid.org/0000-0001-8317-5014

Universidad de Sevilla

Dpto. Física de la Materia Condensada

mbautista@us.es

DOI: http://dx.doi.org/10.12795/9788447231003.033

Pp.: 695-713 


\section{Contexto}

En este capítulo se describe la aplicación de un ciclo de mejora en el aula (CIMA) realizado en la asignatura de Contactología II, que se imparte en tercero del Grado de Òptica y Optometría, y en quinto del Doble Grado de Farmacia y Óptica y Optometría de la Facultad de Farmacia en la Universidad de Sevilla, en donde hay 54 y 19 alumnos matriculados respectivamente durante el curso 2019/20.

Estos alumnos ya han cursado Contactología I, y este ciclo se aplica al final de la asignatura Contactología II, así que ya tienen conocimientos previos de lentes de contacto y valoración de pacientes previa a la adaptación. La finalidad de esta asignatura es profundizar en la selección de pacientes y la metodología de adaptación de diferentes diseños en función de las caracteristicas de la persona a aplicar.

En el momento del curso en el que se aplica el CIMA, los alumnos tienen información sobre las pruebas previas a la adaptación de lentes de contacto y su interpretación. Además, conocen los materiales y diseños de lentes que existen de forma teórica, no la forma en la se comerializan en el mercado actual. En el anterior Ciclo de Mejora se trabajó en el aprendizaje basado en casos clínicos, selección de pacientes y la lente adecuada para cada uno de ellos basándonos en los cálculos teóricos de las mismas (Bautista-Llamas MJ, 2019), pero estos cálculos no siempre se corresponden con los parámetros que nos ofrecen los fabricantes, por lo que ahora los temas sobre los que se van a trabajar son: "Lentes de contacto en el mercado actual" e "Introducción a las lentes de contacto especiales".

Las fechas en las que se desarrolla coinciden con el confinamiento debido al COVID-19 por lo que se erealiza en su totalidad de forma on-line.

Ciclos de Mejora en el Aula (2020). Experiencias de Innovación Docente de la US Esta obra se distribuye con la licencia Creative Commons 


\section{Diseño del CIMA}

\section{Mapa de contenidos}

Como ya se ha comentado, este CIMA tiene como objetivo que los alumnos conozcan las posibilidades que les ofrece el mercado de las lentes de contacto, las caracteristicas de las lentes que se comercializan así como detectar las ventajas de cada una de ellas e identificar las carencias mediante el diseño de su propia lente de contacto. Para ello la pregunta que se les plantea es “¿Qué podemos ofrecer al mercado de las lentes de contacto?". Para dar respuesta a esta pregunta necesitamos conocer: usuarios, materiales, parámetros, diseños y tipos de adaptación que dan lugar a diferentes lentes de contacto, y planificar así la adaptación de las mismas y su seguimento, en casos normales y situaciones especiales, los dos temas ateriromente mencionados sobre los que se van a trabajar (Figura 1) (García-Díaz et al., 2017).

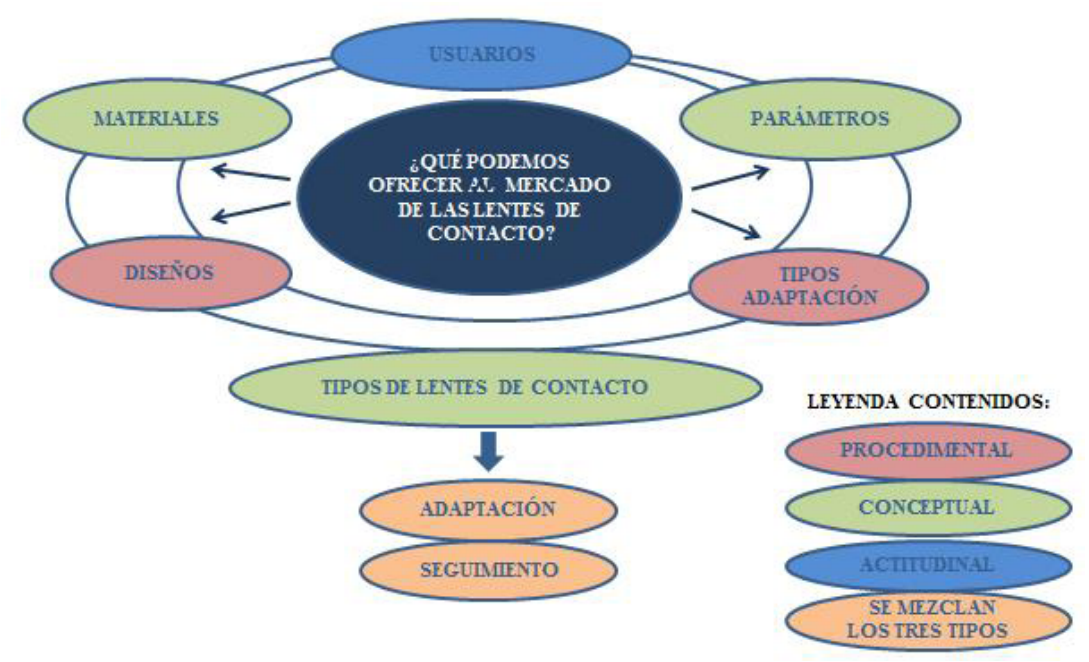

Figura 1. Mapa de contenidos Ciclos de Mejora en el Aula (2020). Experiencias de Innovación Docente de la US Reconocimiento-NoComercial-SinObraDerivada 4.0 Internacional (CC BY-NC-ND 4.0.) 


\section{Modelo metodológico}

Hasta la aplicación de los primeros CIMAs, durante el curso 2018-19, la metodología docente aplicada en la asignatura de Contactología II consiste en una clase expositiva y resolución de problemas. Durante ese curso se cambió este modelo, de forma que son los alumnos los que planteen sus casos, así como su solución y posibles complicaciones en la adapción de lentes de contacto, a la vez de que son capaces de discutirlo con sus compañeros cambiando al una metodología basada en casos y trabajo colaborativo.

Por tanto, en el modelo metodológico posible, me baso en una pregunta sobre el tema que se pretende que aprendan, y al que los alumnos deben dar respuesta a través de la resolución de un problema o caso práctico. Para ello trabajarán en grupos mientras resuelvo de forma individual sus preguntas para ayudales a avanzar moviéndome por la clase.

En la Figura 2 se puede observar el esquema de este modelo, en el que el grueso de la clase lo ocuparía el trabajo de los alumnos. De las soluciones y conclusiones aportadas debería poder extraerse una nueva pregunta que permita avanzar en conocimientos, es decir, un modelo basado en el constructivismo, en el que partiendo de los conocimientos previos de los alumnos lleguemos a un aprendizaje superior (Coll et al., 1993; Cubero, 2005).

Ciclos de Mejora en el Aula (2020). Experiencias de Innovación Docente de la US Esta obra se distribuye con la licencia Creative Commons 


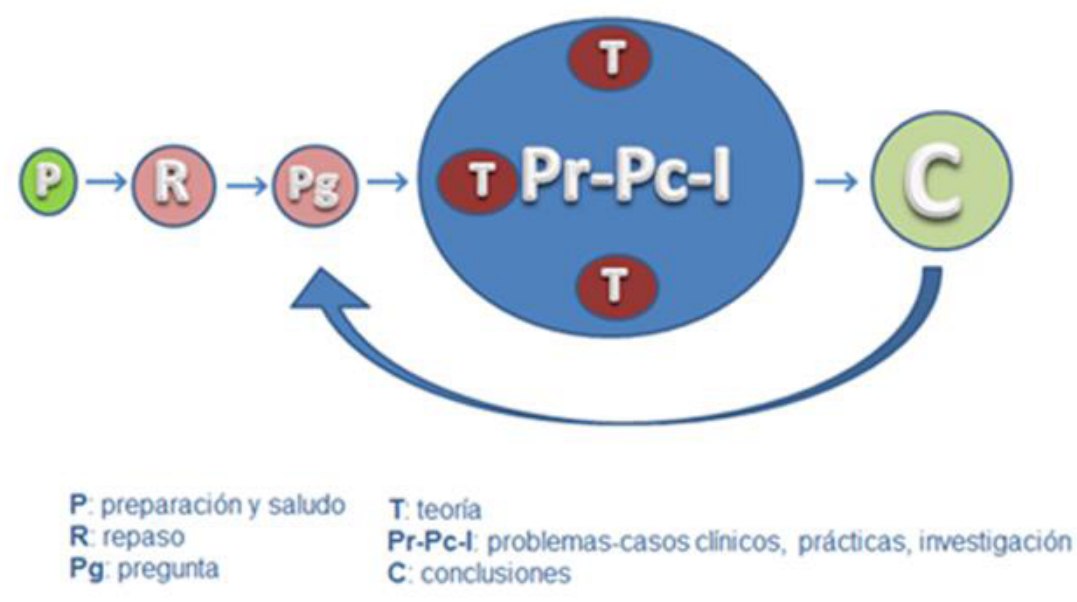

Figura 2. Modelo metodológico posible.

La idea es que sean los propios alumnos quienes planteen preguntas y soluciones según vayan profundizando en la investigación propuesta, acercándose a la práctica en la vida real (Finkel, 2008).

Otro de los retos es que los alumnos sean resolutivos y críticos, discutan su propuesta con sus compañeros, y la defiendan según sus conocimientos.

La forma en que se ha llevado a cabo este modelo metodológico se ha visto afectado por el confinamiento provocado por el COVID-19, lo que implica que las clases se han impartido vía on-line, limitando este sistema la participación de los alumnos, que ellos sean el centro, las discusiones y la supervisión y ayuda en todo momento del profesor al trabajar en clase.

Las clases se han impartido a través de la plataforma Blackboard Collaborate Ultra, y con la misma se han hecho grupos de trabajo para supervisar y ayudar a los alumnos en su investigación, caso o problema.

Ciclos de Mejora en el Aula (2020). Experiencias de Innovación Docente de la US Esta obra se distribuye con la licencia Creative Commons 
La aplicación de este CIMA se llevará a cabo en seis sesiones, en clases de 1 hora y 30 minutos. En las siguientes tablas se describe la secuencia de actividades (Tablas 1, 2, 3 y 4$)$.

\section{Tabla1. Secuencia de actividades en la aplicación del CIMA (sesión 1).}

\begin{tabular}{|c|c|c|c|c|c|}
\hline \multicolumn{6}{|c|}{ IMA (REFID) } \\
\hline FECHA & TIEMPO & ACTIVIDAD & DESARROLLO & FINALIDAD & CONTENIDOS \\
\hline \multirow[t]{5}{*}{$\begin{array}{l}\text { 30-mar.-20 } \\
\text { ON LINE } \\
\text { (Blackboard } \\
\text { Collaborate } \\
\text { Ultra) }\end{array}$} & \multirow[t]{5}{*}{$1 \mathrm{~h}$ y $30 \mathrm{~min}$} & $\begin{array}{l}\text { Presentar el } \\
\text { ejercicio. }\end{array}$ & $\begin{array}{l}\text { Trabajo en grupo } \\
\text { asumiendo el rol de } \\
\text { fabricantes de lentes de } \\
\text { contacto. Deben } \\
\text { investigar qué tipos de } \\
\text { materiales y diseños } \\
\text { existen en el mercado, a } \\
\text { qué tipo de pacientes se } \\
\text { dirigen y diseñar la suya. }\end{array}$ & $\begin{array}{l}\text { Conocer la } \\
\text { secuencia de } \\
\text { trabajo. }\end{array}$ & \multirow[t]{5}{*}{$\begin{array}{l}\text { Características de } \\
\text { las lentes de } \\
\text { contacto que } \\
\text { existen en el } \\
\text { mercado. }\end{array}$} \\
\hline & & $\begin{array}{l}\text { Resolver } \\
\text { dudas. }\end{array}$ & $\begin{array}{l}\text { Responder a las } \\
\text { preguntas de los alumnos } \\
\text { vía mail. }\end{array}$ & & \\
\hline & & $\begin{array}{l}\text { Responder } \\
\text { cuestionario } \\
\text { inicial. }\end{array}$ & $\begin{array}{l}\text { Cada alumno responde al } \\
\text { cuestionario de forma } \\
\text { corta y clara a través de } \\
\text { la plataforma Google } \\
\text { forms. }\end{array}$ & $\begin{array}{l}\text { Determinar el } \\
\text { nivel de } \\
\text { conocimientos } \\
\text { de los } \\
\text { alumnos. }\end{array}$ & \\
\hline & & $\begin{array}{l}\text { Crear grupos } \\
\text { de trabajo. }\end{array}$ & $\begin{array}{l}\text { Se hacen grupos de } \\
\text { trabajo. Plataformas para } \\
\text { trabajar: Skype, } \\
\text { Whatsapp, Messenger, } \\
\text { Go to webinar, Go to } \\
\text { meeting o Blackboard } \\
\text { Collaborate Ultra. }\end{array}$ & $\begin{array}{l}\text { Con el trabajo } \\
\text { en grupo se } \\
\text { trata de } \\
\text { fomentar el } \\
\text { intercambio } \\
\text { de opiniones. }\end{array}$ & \\
\hline & & $\begin{array}{l}\text { Trabajar en } \\
\text { grupo. }\end{array}$ & $\begin{array}{l}\text { Deberán dedicar al menos } \\
\text { una hora a la } \\
\text { investigación para poder } \\
\text { responder a las preguntas } \\
\text { que iniciarán la siguiente } \\
\text { sesión. }\end{array}$ & $\begin{array}{l}\text { Avanzar en } \\
\text { conocimientos }\end{array}$ & \\
\hline
\end{tabular}

Ciclos de Mejora en el Aula (2020). Experiencias de Innovación Docente de la US Esta obra se distribuye con la licencia Creative Commons 
Tabla 2. Secuencia de actividades en la aplicación del CIMA (sesión 2).

\begin{tabular}{|c|c|c|c|c|c|}
\hline \multicolumn{6}{|c|}{ CIMA (REFID) } \\
\hline FECHA & TIEMPO & ACTIVIDAD & DESARROLLO & FINALIDAD & CONTENIDOS \\
\hline \multirow{5}{*}{$\begin{array}{l}\text { 13-abr.-20 } \\
\text { y 20-abr-20 } \\
\text { ON LINE } \\
\text { (Blackboard } \\
\text { Collaborate } \\
\text { Ultra) }\end{array}$} & $5 \mathrm{~min}$ & $\begin{array}{l}\text { Repasar la clase } \\
\text { anterior y poner en } \\
\text { situación para seguir } \\
\text { trabajando hoy. }\end{array}$ & $\begin{array}{l}\text { Breve explicación } \\
\text { sobre la clase } \\
\text { anterior. }\end{array}$ & $\begin{array}{l}\text { Poner en } \\
\text { situación a los } \\
\text { alumnos. }\end{array}$ & \multirow{5}{*}{$\begin{array}{l}\text { Características de } \\
\text { las lentes de } \\
\text { contacto que } \\
\text { existen en el } \\
\text { mercado. }\end{array}$} \\
\hline & $10 \mathrm{~min}$ & $\begin{array}{l}\text { Kahoot sobre la } \\
\text { sesión anterior. }\end{array}$ & $\begin{array}{l}\text { Enlace al } \\
\text { cuestionario en } \\
\text { enseñanza virtual. }\end{array}$ & $\begin{array}{l}\text { Conocer la } \\
\text { investigación. }\end{array}$ & \\
\hline & $30 \mathrm{~min}$ & $\begin{array}{l}\text { Análisis de las } \\
\text { respuestas obtenidas, } \\
\text { estado de la } \\
\text { investigación y dudas } \\
\text { de los alumnos. }\end{array}$ & $\begin{array}{l}\text { Utilización del chat } \\
\text { dentro de la } \\
\text { herramienta } \\
\text { Blackboard } \\
\text { Collaborate Ultra. }\end{array}$ & $\begin{array}{l}\text { Ayudar a los } \\
\text { alumnos en su } \\
\text { trabajo de } \\
\text { investigación. }\end{array}$ & \\
\hline & $40 \mathrm{~min}$ & $\begin{array}{l}\text { Creación de grupos } \\
\text { dentro de Blackboard } \\
\text { Collaborate Ultra. }\end{array}$ & $\begin{array}{l}\text { Los alumnos } \\
\text { trabajan en grupo } \\
\text { los conceptos que } \\
\text { hemos aclarado en } \\
\text { clase. }\end{array}$ & $\begin{array}{l}\text { Trabajo en } \\
\text { grupo. }\end{array}$ & \\
\hline & $5 \mathrm{~min}$ & $\begin{array}{l}\text { Dar instrucciones } \\
\text { para la siguiente } \\
\text { clase. }\end{array}$ & $\begin{array}{l}\text { En la siguiente clase } \\
\text { se expondrán los } \\
\text { resultados. }\end{array}$ & $\begin{array}{l}\text { Desarrollo de } \\
\text { la siguiente } \\
\text { clase. }\end{array}$ & \\
\hline
\end{tabular}

Tabla 3. Secuencia de actividades en la aplicación del CIMA (sesión 3).

\begin{tabular}{|c|c|c|c|c|c|}
\hline \multicolumn{6}{|c|}{ CIMA (REFID) } \\
\hline FECHA & TIEMPO & ACTIVIDAD & DESARROLLO & FINALIDAD & CONTENIDOS \\
\hline \multirow{5}{*}{$\begin{array}{l}\text { 20-abr.-20 } \\
\text { ON LINE } \\
\text { (Blackboard } \\
\text { Collaborate } \\
\text { Ultra) }\end{array}$} & $5 \mathrm{~min}$ & $\begin{array}{l}\text { Repasar la clase } \\
\text { anterior }\end{array}$ & $\begin{array}{l}\text { Breve explicación } \\
\text { sobre la clase anterior. }\end{array}$ & $\begin{array}{l}\text { Poner en } \\
\text { situación a los } \\
\text { alumnos }\end{array}$ & \multirow{2}{*}{$\begin{array}{l}\text { Características de } \\
\text { las lentes de } \\
\text { contacto que } \\
\text { existen en el } \\
\text { mercado }\end{array}$} \\
\hline & $10 \mathrm{~min}$ & $\begin{array}{l}\text { Kahoot sobre la } \\
\text { sesión anterior. }\end{array}$ & $\begin{array}{l}\text { En la plataforma de } \\
\text { enseñanza virtual se } \\
\text { cuelga enlace al } \\
\text { cuestionario. }\end{array}$ & $\begin{array}{l}\text { Conocer el } \\
\text { desarrollo de la } \\
\text { investigación }\end{array}$ & \\
\hline & $30 \mathrm{~min}$ & $\begin{array}{l}\text { Análisis de las } \\
\text { respuestas } \\
\text { obtenidas }\end{array}$ & $\begin{array}{l}\text { Utilización del chat } \\
\text { dentro de la } \\
\text { herramienta } \\
\text { Blackboard } \\
\text { Collaborate Ultra }\end{array}$ & $\begin{array}{l}\text { Ayudar a los } \\
\text { alumnos en su } \\
\text { trabajo de } \\
\text { investigación. }\end{array}$ & \\
\hline & $40 \mathrm{~min}$ & $\begin{array}{l}\text { Trabajo en grupo } \\
\text { dentro } \\
\text { Blackboard } \\
\text { Collaborate } \\
\text { Ultra. }\end{array}$ & $\begin{array}{l}\text { Los alumnos trabajan } \\
\text { en grupo los } \\
\text { conceptos que hemos } \\
\text { acaparado en clase. }\end{array}$ & $\begin{array}{l}\text { Trabajo en } \\
\text { grupo }\end{array}$ & \\
\hline & $5 \mathrm{~min}$ & $\begin{array}{l}\text { Explicar } \\
\text { siguiente } \\
\text { actividad. }\end{array}$ & $\begin{array}{l}\text { Trabajar en grupo y } \\
\text { establecer un plan de } \\
\text { seguimiento según la } \\
\text { lente de contacto } \\
\text { adaptada. }\end{array}$ & & \\
\hline
\end{tabular}



(sesiones 4 y 5 ).

\begin{tabular}{|l|c|l|l|l|l|}
\hline FECHA & TIEMPO & ACTIVIDAD & DESARROLLO & FINALIDAD & \multicolumn{1}{|c|}{ CONTENIDOS } \\
\hline $\begin{array}{l}\text { 4-may.-20 } \\
\text { y 11-may-20 }\end{array}$ & 5 min & $\begin{array}{l}\text { Repasar la clase } \\
\text { anterior. }\end{array}$ & $\begin{array}{l}\text { Breve explicación } \\
\text { sobre la clase anterior. }\end{array}$ & $\begin{array}{l}\text { Poner en } \\
\text { situación a los } \\
\text { alumnos }\end{array}$ & $\begin{array}{l}\text { Características de } \\
\text { las lentes de } \\
\text { contacto que } \\
\text { existen en el } \\
\text { mercado. }\end{array}$ \\
\hline ON LINE & (Blackboard \\
Collaborate \\
Ultra)
\end{tabular}




\section{Cuestionario inicial y final}

Para evaluar la efectividad del CIMA, y siguiendo las directrices recogidas en Rivero y Porlán (2017), se elabora un cuestionario para pasarlo a los estudiantes antes y después del ciclo. Estas son las preguntas formuladas a los estudiantes:

1. Tengo ojo seco, ¿qué material me iría mejor para soportar durante más tiempo mis lentes de contacto?

2. Mi madre tiene miopía y presbicia, quiere llevar lentes de contacto, pero mi tía le ha dicho que provocan muchos destellos, ¿es verdad? ¿Por qué? ¿Existe alguna lente con la que no aparezcan?

3. ¿Para graduaciones elevadas todas las lentes de contacto ofrecen la misma calidad visual?

4. Me operé de miopía hace 5 años y vuelvo a tener graduación, miopía y astigmatismo, ¿puedo usar lentes de contacto? ¿De que tipo?

5. A mi hermano le acaban de diagnosticar queratocono, ¿puede usar lentes de contacto? ¿Cuáles le recomendarías?

Las respuestas son clasificadas en función de los diferentes niveles de aprendizaje identificados y descritos en la Tabla3. Estos niveles se han establecido en función de si los alumnos optaban por respuestas simples o iban añadiendo información detallada y justificada a las mismas.

Ciclos de Mejora en el Aula (2020). Experiencias de Innovación Docente de la US Esta obra se distribuye con la licencia Creative Commons 
Tabla 3. Clasificación de las respuestas obtenidas en el cuestionario.

\section{CUESTIONARIO INICIAL Y FINAL}

\begin{tabular}{|c|c|c|c|c|}
\hline $\begin{array}{l}\text { PREGUNTA/NIVEL } \\
\text { ES }\end{array}$ & $\mathbf{A}$ & B & C & D \\
\hline $\begin{array}{l}\text { Tengo ojo seco, ¿qué } \\
\text { material me iría mejor } \\
\text { para soportar durante } \\
\text { más tiempo mis lentes } \\
\text { de contacto? }\end{array}$ & $\begin{array}{l}\text { No } \\
\text { puedes usar } \\
\text { lentes de } \\
\text { contacto }\end{array}$ & $\begin{array}{l}\text { Cualquiera } \\
+ \\
\text { Lágrima } \\
\text { artificial }\end{array}$ & $\begin{array}{l}\text { Diferencia entre } \\
\text { materiales y } \\
\text { recomienda uno. }\end{array}$ & $\begin{array}{c}\text { Diferencia entre } \\
\text { materiales de lentes } \\
\text { de contacto, } \\
\text { tratamientos } \\
\text { adicionales al } \\
\text { material de la lente } \\
\text { y tipos de ojo seco. }\end{array}$ \\
\hline $\begin{array}{l}\text { Mi madre tiene } \\
\text { miopía y presbicia, } \\
\text { quiere llevar lentes de } \\
\text { contacto, pero mi tía le } \\
\text { ha dicho que provocan } \\
\text { muchos destellos, ¿es } \\
\text { verdad? ¿Por qué? } \\
\text { ¿Existe alguna lente con } \\
\text { la que no aparezcan? }\end{array}$ & $\begin{array}{l}\text { No, no } \\
\text { explica }\end{array}$ & $\begin{array}{l}\text { Sí, explica } \\
\text { brevemente. } \\
\text { Respuestas no } \\
\text { muy acertadas. }\end{array}$ & $\begin{array}{c}\text { Sí, explica } \\
\text { razonadamente } \\
\text { pero no recomienda } \\
\text { alternativas. }\end{array}$ & $\begin{array}{c}\text { Sí, explica } \\
\text { razonadamente y } \\
\text { recomienda } \\
\text { alternativas. }\end{array}$ \\
\hline $\begin{array}{l}\text { ¿Para graduaciones } \\
\text { elevadas todas las lentes } \\
\text { de contacto ofrecen la } \\
\text { misma calidad visual? }\end{array}$ & $\mathrm{Si}$ & No & $\begin{array}{l}\text { No, explica } \\
\text { brevemente. }\end{array}$ & $\begin{array}{c}\text { No, razona } \\
\text { porque y cuáles } \\
\text { serían las } \\
\text { mejores. }\end{array}$ \\
\hline $\begin{array}{c}\text { Me operé de miopía } \\
\text { hace } 5 \text { años y vuelvo a } \\
\text { tener graduación, } \\
\text { miopía y astigmatismo, } \\
\text { ¿puedo usar lentes de } \\
\text { contacto? ¿De que tipo? }\end{array}$ & $\begin{array}{c}\text { No } \\
\text { sabe/no } \\
\text { contesta }\end{array}$ & $\begin{array}{c}\text { Si. } \\
\text { Sin } \\
\text { explicaciones. }\end{array}$ & $\begin{array}{c}\mathrm{Si} \text {, explica } \\
\text { brevemente cuales. }\end{array}$ & $\begin{array}{c}\text { Sí, explica } \\
\text { razonadamente las } \\
\text { alternativas. }\end{array}$ \\
\hline $\begin{array}{c}\text { A mi hermano le } \\
\text { acaban de diagnosticar } \\
\text { queratocono, ¿puede } \\
\text { usar lentes de contacto? } \\
\text { ¿Cuáles le } \\
\text { recomendarías? }\end{array}$ & No & $\begin{array}{l}\text { Sí, no sabe } \\
\text { cuáles. }\end{array}$ & $\begin{array}{c}\mathrm{Si} \text {, tiene } \\
\text { conocimientos pero } \\
\text { no profundiza. }\end{array}$ & $\begin{array}{l}\text { Si, describe } \\
\text { características } \\
\text { (parámetros } \\
\text { oculares y } \\
\text { refracción) en } \\
\text { función del grado } \\
\text { de la patología. }\end{array}$ \\
\hline
\end{tabular}

\section{Aplicación del CIMA}

Comienza el CIMA con la explicación del proyecto que vamos a desarrollar durante las siguientes clases, un trabajo en grupo en el que se convertirán en "fabricantes de lentes de contacto" para poder profundizar en el mercado, encontrar los puntos fuertes y débiles, y realizar sus propuestas. Después los expondrán y debatirán con el resto de la clase para aclarar conceptos.

Ciclos de Mejora en el Aula (2020). Experiencias de Innovación Docente de la US (C) Esta obra se distribuye con la licencia Creative Commons Reconocimiento-NoComercial-SinObraDerivada Internacional (CC BY-NC-ND 4.0.) 
Para motivarles extrínsecamente les explico que entre los casos expuestos se elegirán los más trabajados y completos y obtendrán puntuación extra en el examen parcial. Tomo esta medida para motivar a los sectores menos participativos y que presentan menos interés en general.

Antes de que se pongan a trabajar contestan vía Google forms al cuestionario sobre el tema para valorar los posibles saltos en su aprendizaje.

Al trabajar de forma on line hemos utilizado las clases de forma diferente, normalmente se formarían grupos y la profesora atiende las demandas de cada grupo mientras trabajan sobre el tema, en esta ocasión hemos utilizado las tres primeras sesiones para organizar el trabajo, crear objetivos y responder dudas hasta ese objetivo. Se intentó trabajar en grupos independientes durante las sesiones y que la profesora se conectará en la misma sesión con cada uno de ellos, simulando la opción presencial, pero el resultado fue caótico, por lo que se optó en la tercera sesión por aclarar conceptos de forma general y corregir con tutorías independientes en diferentes fechas y horas con cada grupo para ayudarles a llegar al objetivo. El hecho de no estar familiarizados con la plataforma y con la comunicación a través de una pantalla creo que hace que el trabajo no sea tan fluido como cuando lo realizamos en el aula.

Cada una de estas semanas se les ha pasado un cuestionario (Kahoot) para valorar la implicación en el proyecto, obteniendo resultados muy favorables en general, lo que indica que la implicación, a pesar de que las herramientas y formato no era el más adecuado, de los alumnos ha sido buena.

En las sesiones restantes, 4, 5 y 6, se exponen todos los trabajos. Se dividen, con alguna excepción, entre lentes de contacto multifocales y especiales para usuarios de 
dispositivos electrónicos y con ojo seco, su trabajo de investigación es bueno, porque son los dos grandes grupos que copan el mercado actualmente.

Nos vamos ajustando a los tiempos planificados y los diseños planteados nos dan pie a discutir sobre cómo funciona la prescripción de lentes de contacto en la realidad, con que van a trabajar.

En general todos los trabajos son buenos, y me encuentro con uno que no ha diseñado una lente, si no que ha creado un portfolio con la que consideran mejor lente en función del reemplazo que tiene y diseño. Con este trabajo cerramos las sesiones y me ayuda a hacer un resumen. Todo un acierto puesto que fue la sesión en la que más alumnos participaron. La elaboración de un portfolio, bien sea como en este caso, de un solo tema o trabajo propuesto por el profesor, o como evaluación continua de una asignatura, me parece una de las mejores formas de valorar el trabajo y esfuerzo realizado por un alumno, así como una muy buena forma de aprender durante la duración de una asignatura avanzando en conocimientos sin dejar nada atrás.

\section{Resultados del cuestionario inicial y final. Escaleras de aprendizaje}

El cuestionario recogido en la Tabla 3 fue respondido por los 73 alumnos matriculados en la asignatura, prácticamente el $100 \%$ de la clase, que ha sido lo bueno de la situación propiciada por el COVID-19.

Analizando las respuestas, en la pregunta 1, "Tengo ojo seco, ¿qué material me iría mejor para soportar durante más tiempo mis lentes de contacto?" es donde he encontrado el nivel más bajo en las respuestas iniciales, creo 
que porque siempre tratamos el ojo seco como un obstáculo para el uso de lentes de contacto (Figura 3). Aquí queda claro que en clase normalmente no damos a conocer este mercado, lleno de alternativas en materiales y tratamientos para los mismos con el fin de evitar la evaporación de la lágrima y aumentar el confort del usuario. Tras realizar el trabajo de investigación propuesto, estos porcentajes pasan a los escalones más altos. Fue una grata sorpresa para los alumnos encontrarse con una gran cantidad de posibilidades que ofrecer a los usuarios que padecen esta patología.

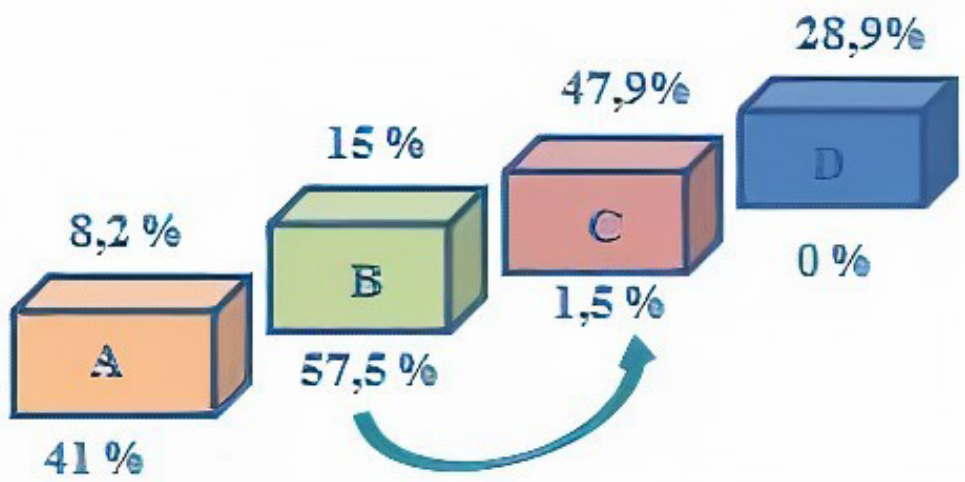

Figura 3. Escalera de aprendizaje para la pregunta 1(las cifras inferiores indican los valores de la encuesta inicial y la superior la final).

Para la pregunta dos, la respuesta sobre el uso de las lentes de contacto para présbitas y los posibles destellos que pueden observar aquellos pacientes que las usan, el mayor porcentaje de respuestas se encuentra en el segundo escalón y pasan al tercero en el cuestionario final (Figura 4). En esta ocasión los porcentajes se invierten, pasando los de los dos escalones más bajos a los más altos, aunque la gran mayoría sigue sin poder explicar de forma clara las alternativas, en cuanto a diseño de lentes multifocales se refiere, que existen.

Ciclos de Mejora en el Aula (2020). Experiencias de Innovación Docente de la US Esta obra se distribuye con la licencia Creative Commons 

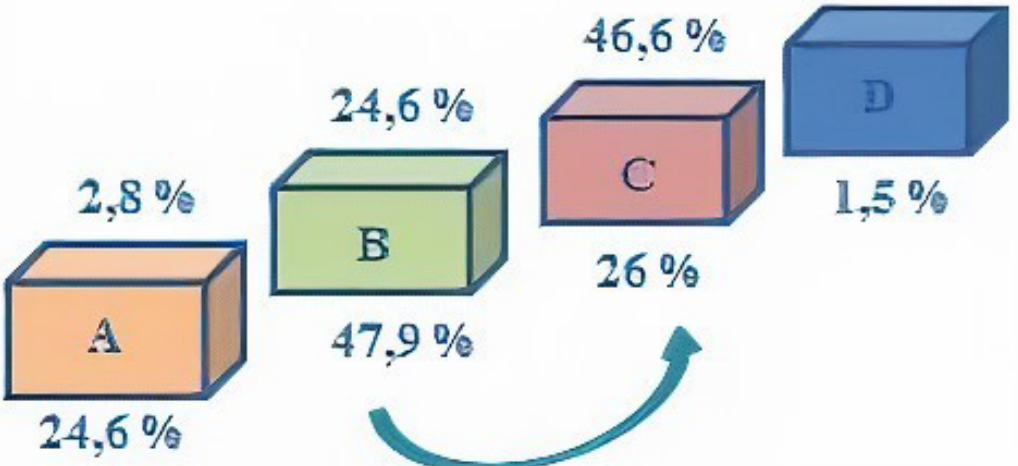

$26 \%$

$47,9 \%$

En la pregunta 3 es donde observamos un mayor conocimento previo del tema, saben que podemos compensar todo tipo de ametropías, aunque no tienen claro cual es la mejor alternativa para cada una de ellas. Sin embargo es en el tema en el que los conceptos se aclaran más, y la mitad de la clase alcanza el nivel máximo en cuanto al conocimiento del tema se refiere (Figura 5).

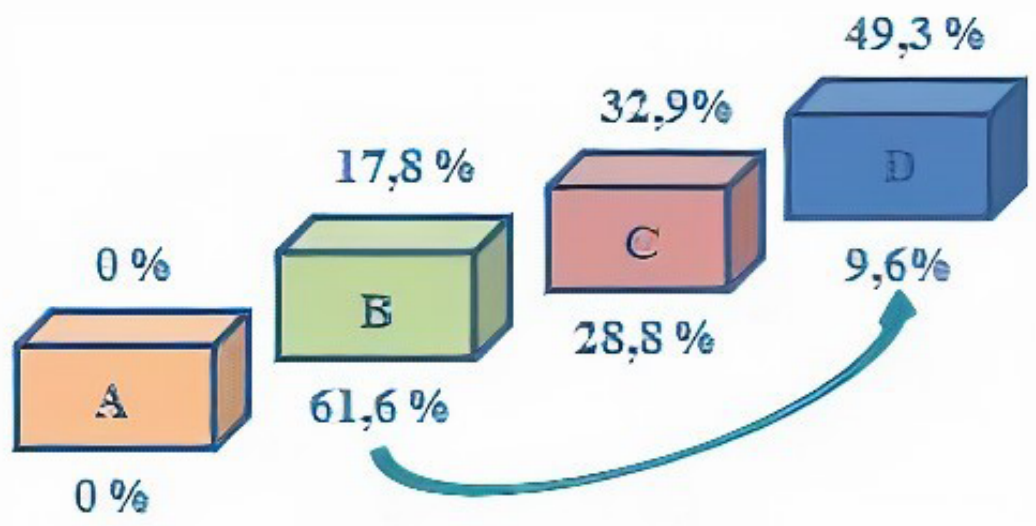

Figura 5. Escalera de aprendizaje para la pregunta 3.

Ciclos de Mejora en el Aula (2020). Experiencias de Innovación Docente de la US Esta obra se distribuye con la licencia Creative Commons 
En la cuarta pregunta nos acercamos a las lentes de contacto especiales, post cirugía en este caso, aquí no espero que tengan ningún conocimento previo del tema, así que que la mayoría sepa al menos que se pueden usar, ya me parece suficiente (Figura 6). Es sorprendente como casi el $40 \%$ de la clase ha sido capaz de saltar hasta el escalón más alto del conocimiento propuesto del tema. Esto me confirma que han realizado con interés la investigación, porque son lentes poco comunes, con poca publicidad y que ocupan un papel secundario, con lo cual es más dificil acceder a la información sobre las mismas.

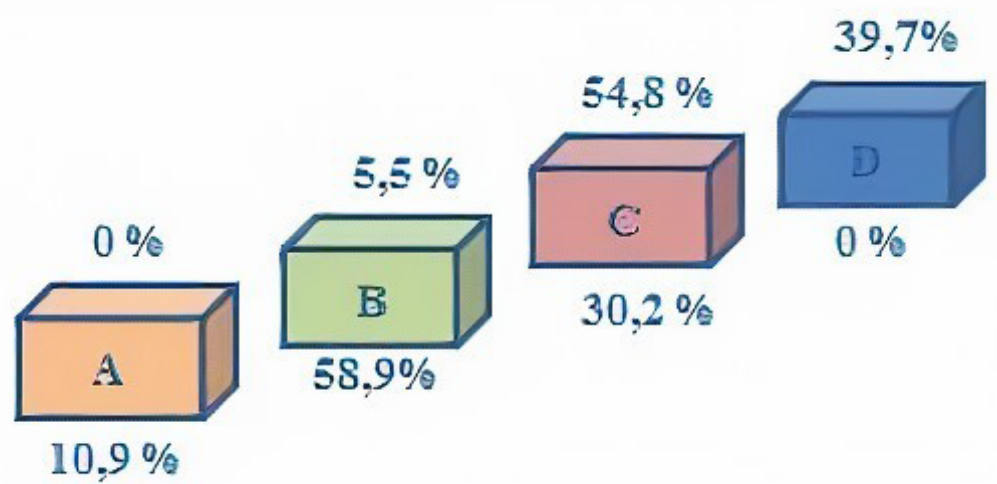

Figura 6. Escalera de aprendizaje para la pregunta 4.

Por último, y siguiendo con el tema de lentes especiales, se pregunta por lentes para una patología en concreto, el Queratocono. En este caso también se aprecia una evolución en los conocimientos sobre el tema (Figura 7). Inicialmente tenían claro que se podían usar lentes de contacto, pero al final casi todos se acercan al tipo de lentes que se usan y un tercio de la clase sabe relaccionar el grado de la patología con la adaptación que necesitará el paciente.

Con las dos últimas preguntas me aseguro un nivel de conocimientos para la asignatura que complementa a esta

Ciclos de Mejora en el Aula (2020). Experiencias de Innovación Docente de la US Esta obra se distribuye con la licencia Creative Commons 
en el próximo curso y en la que se tratan casos concretos sobre patologías, el conocimiento del mercado de las mismas hará que se profundice mucho más en la prescripción que se haga en estos casos.

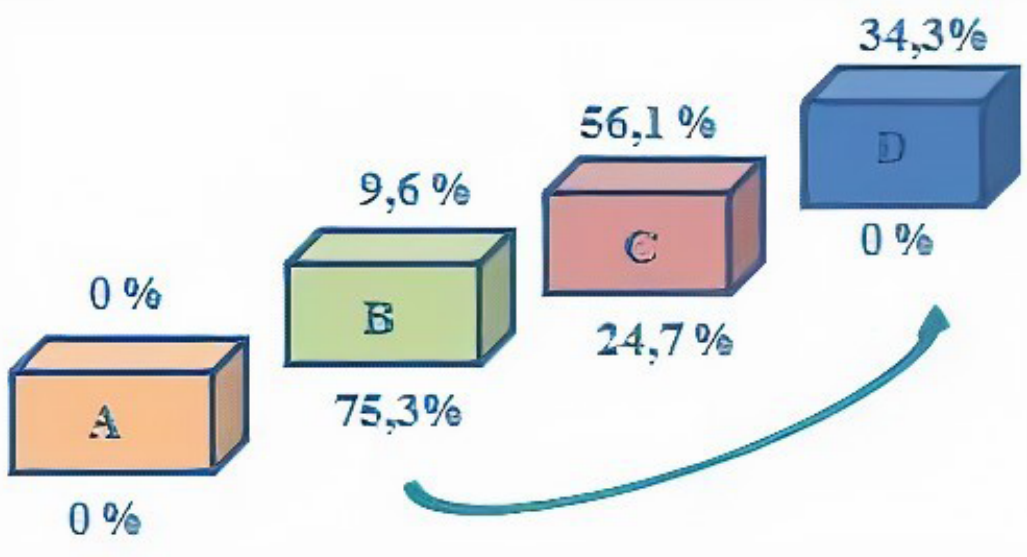

Figura 7. Escalera de aprendizaje para la pregunta 5.

Tras comparar las respuestas del cuestionario inicial con las del final se aprecia que en la mayoría de los alumnos existe una evolución en sus respuestas, aunque siempre existen excepciones, alumnos con menos interés y que se mantienen en los niveles iniciales. En general, y contra pronóstico por la situación excepcional en la que nos encontramos en la aplicación de este ciclo de mejora, la evolución ha sido mejor que la esperada.

\section{Evaluación del CIMA}

Los resultados obtenidos en el análisis del cuestionario y reflejados en las escaleras de aprendizaje demuestran que el diseño ha cumplido con su cometido, ya que han mejorado los resultados con respecto al cuestionario inicial, aunque la situación en la que se ha trabajado no haya sido la más apropiada para lo que se pretendía.

Ciclos de Mejora en el Aula (2020). Experiencias de Innovación Docente de la US Esta obra se distribuye con la licencia Creative Commons 
Si comparo los resultados con el CIMA anterior me he encontrado mucho más cómoda a la hora de elaborar los cuestionarios o las actividades, así como de dirigir a los alumnos a los objetivos que me había planteado.

Para mí es algo fundamental que los alumnos participen en clase, y ese el objetivo de este proyecto de innovación docente, que ellos se conviertan en el centro, pero la situación vivida este curso, desde mi punto de vista, nos ha impedido alcanzar ese objetivo. Comunicarnos a través de una pantalla de ordenador no favorece la participación de los alumnos, al contrario, lo tienen mucho más fácil para esconderse, ni siquiera el "anonimato" de escribir a través de un chat hace que algunos, por no decir la gran mayoría, participe, y ese es el punto negro en mi CIMA. No tengo feedback de los alumnos, excepto por el cuestionario, y de los 10-15 que han participado de forma activa, el resto no puedo saber ni siquiera si han estado escuchando. Por los resultados puedo concluir que han realizado su parte del proyecto dentro de los grupos a los que pertenecían, por lo tanto han aumentado sus conocimientos, pero para mí hemos perdido el segundo objetivo, ser crítico con lo aprendido y discutirlo con los compañeros, para eso, necesitamos una clase presencial y que la interacción sea entre todas las partes (Bain, 2007).

Por otro lado, debo valorar de forma positiva la implicación en los trabajos de los alumnos, aunque esta no se vea reflejada en la discusión de los mismos, así como el agradecimiento que demuestran ante el aprendizaje virtual no conocido en las aulas.

Al igual que en la aplicación del anterior CIMA el mapa de contenidos, junto con el diseño de la secuencia de actividades, de forma que se pueda manejar mejor el hilo conductor de una clase, manteniendo la atención y desarrollando trabajos dentro y fuera de la misma y que 
implique más participación e independencia en el aprendizaje, siguen siendo para mí el valor añadido a mi metodología de trabajo. Además, empiezo a considerar las preguntas clave y los escalones de aprendizaje una herramienta fundamental para la evaluación de lo ocurrido en las clases.

Resumiendo, la aplicación del CIMA, dentro del Programa de Formación e Innovación Docente del Profesorado 2020 (FIDOP) en la fase de permanencia (REFID), ha supuesto un gran reto por intentar llevar la innovación docente a los alumnos a través de la enseñanza virtual, una plataforma que para mí nos aleja del objetivo en mi caso, que es la participación activa de los alumnos en las clases, aunque no ha influido en el proyecto de investigación propuesto y que han superado con creces.

Ciclos de Mejora en el Aula (2020). Experiencias de Innovación Docente de la US Esta obra se distribuye con la licencia Creative Commons 
Palabras Clave: Contactología II, Grado en Óptica y Optometría, Doble grado en Farmacia Óptica y Optometria, docencia universitaria, experimentación docente universitaria.

Keywords: Contactology II, Degree on Optics and Optometry, Double Degree on Pharmacy and Optics and Optometry, university teaching, university teaching experimentation.

\section{Referencias bibliográficas}

Bain K. (2007). Lo que hacen los mejores profesores universitarios. Valencia: Publicacions de la Universitat de Valéncia.

Bautista-Llamas MJ. Aprendizaje basado en el planteamiento de casos clínicos en Contactología. En R. Porlán y E. Navarro-Medina (Coord.), Ciclos de mejora en el aula. Año 2019. Experiencias de innovación docente de la Universidad de Sevilla, pp. 267-287. Sevilla: Servicio de Publicaciones de la Universidad de Sevilla. https:// dx.doi.org/10.12795/9788447221912.011

Coll, C. et al. (1993). El constructivismo en el aula. Barcelona: Graó.

Cubero, R. (2005). Perspectivas constructivistas. La intersección entre el significado, la interacción y el discurso. Barcelona: Graó.

Finkel D. (2008). Dar clase con la boca cerrada. Valencia: Publicacions de la Universitat de Valéncia.

García Díaz E., Porlán R. y Navarro E. (2017). Los fines y los contenidos de la enseñanza. En R. Porlán (Coord.), Enseñanza Universitaria. Cómo mejorarla, pp. 55-72. Madrid: Morata.

Ciclos de Mejora en el Aula (2020). Experiencias de Innovación Docente de la US Esta obra se distribuye con la licencia Creative Commons 